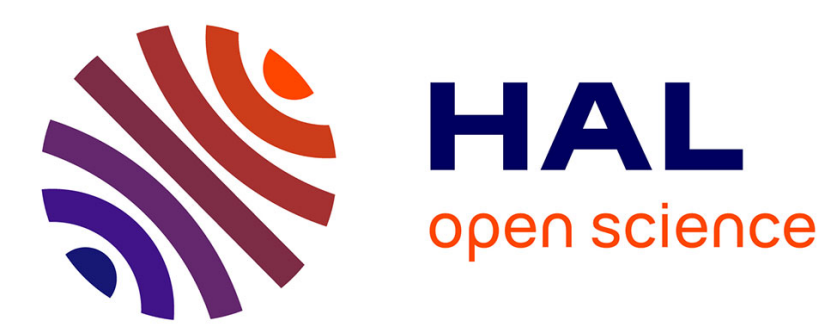

\title{
Elucidating Curvature-Capacitance Relationships in Carbon-Based Supercapacitors
}

\author{
Jannes Seebeck, Céline Merlet, Robert Meissner
}

\section{To cite this version:}

Jannes Seebeck, Céline Merlet, Robert Meissner. Elucidating Curvature-Capacitance Relationships in Carbon-Based Supercapacitors. Physical Review Letters, 2022, 128 (8), pp.086001. 10.1103/physrevlett.128.086001 . hal-03588862

\section{HAL Id: hal-03588862 \\ https://hal.science/hal-03588862}

Submitted on 25 Feb 2022

HAL is a multi-disciplinary open access archive for the deposit and dissemination of scientific research documents, whether they are published or not. The documents may come from teaching and research institutions in France or abroad, or from public or private research centers.
L'archive ouverte pluridisciplinaire HAL, est destinée au dépôt et à la diffusion de documents scientifiques de niveau recherche, publiés ou non, émanant des établissements d'enseignement et de recherche français ou étrangers, des laboratoires publics ou privés. 


\title{
Elucidating Curvature-Capacitance Relationships in Carbon-Based Supercapacitors
}

\author{
Jannes Seebeck \\ Institute of Polymers and Composites, Hamburg University of Technology, 21073 Hamburg, Germany \\ Céline Merlet \\ CIRIMAT, Université de Toulouse, CNRS, Université Toulouse 3 - Paul Sabatier, \\ 118 Route de Narbonne, 31062 Toulouse cedex 9 - France \\ and Réseau sur le Stockage Electrochimique de l'Energie (RS2E), Fédération de Recherche CNRS 3459, \\ HUB de l'Energie, Rue Baudelocque, 80039 Amiens, France \\ Robert H. Meißner®* \\ Institute of Polymers and Composites, Hamburg University of Technology, 21073 Hamburg, Germany \\ and Helmholtz-Zentrum Hereon, Institute of Surface Science, 21502 Geesthacht, Germany
}

(Received 22 July 2021; revised 28 January 2022; accepted 1 February 2022; published 24 February 2022)

\begin{abstract}
Nanoscale surface curvatures, either convex or concave, strongly influence the charging behavior of supercapacitors. Rationalizing individual influences of electrode atoms to the capacitance is possible by interpreting distinct elements of the charge-charge covariance matrix derived from individual charge variations of the electrode atoms. An ionic liquid solvated in acetonitrile and confined between two electrodes, each consisting of three undulated graphene layers, serves as a demonstrator to illustrate pronounced and nontrivial features of the capacitance with respect to the electrode curvature. In addition, the applied voltage determines whether a convex or concave surface contributes to increased capacitance. While at lower voltages capacitance variations are in general correlated with ion number density variations in the double layer formed in the concave region of the electrode, for certain electrode designs a surprisingly strong contribution of the convex part to the differential capacitance is found both at higher and lower voltages.
\end{abstract}

DOI: 10.1103/PhysRevLett.128.086001

Since energy storage in supercapacitors does not happen through redox reactions but by the formation of a thin interfacial region between electrode and electrolyte, attainable capacitances are greatly influenced by the explicit structure and dynamics of this electric double layer (EDL). The discovery of an anomalously high capacitance in microporous carbons has led to numerous research activities aimed at deciphering the underlying molecular mechanisms [1-7]. Beyond the sheer increase of the electrode surface area facilitated by microporous carbons-effectively increasing the capacitance - the specific shape of the electrodes, such as pore sizes and curvatures, affects the structure and composition of the EDL and thus the capacitance [8-15].

From a physical perspective, the charge on an electrode in contact with an electrolyte and maintained at a constant electric potential is subject to thermal fluctuations that encode information about microscopic interfacial processes [8]. Force field based simulations in a constant potential (CONP) ensemble have become an increasingly powerful tool to study these interfacial processes computationally [16-19]. Rather than a simple and often insufficient use of a homogeneously distributed constant charge, in a CONP approach [20-22] charges of individual electrode atoms are treated as additional degrees of freedom that vary in response to the microscopic configuration of the electrolyte at a given external applied potential. This corresponds to a classical treatment of the charge distribution in an electrode corresponding to a given applied potential and nearby electrolyte configuration, and assuming that the electrode material is a perfect metal. While experimentally, the EDL of ionic liquids has been studied by $\mathrm{x}$-ray electron spectroscopy [23], quasielastic neutron scattering [24], nuclear magnetic resonance spectroscopy [25], scanning tunneling microscopy [26-28], and dynamic [29,30] or static [31,32] atomic force microscopy, CONP methods ultimately allow for the simulation of a variety of electrochemical systems with complex geometries, e.g., carbide-derived carbons in contact with a variety of electrolytes, such as aqueous or organic solutions, room temperature ionic liquids, and salts dissolved in water [33-37].

Previous simulations investigated charge fluctuations on the electrode using a histogram reweighting approach to capture more accurately free-energy differences and capacitances or number densities as a function of voltage $[8,16,38-40]$. This allowed for the identification of a voltage-induced phase transition in the first adsorbed layer of an ionic liquid at room temperature on a flat metallic electrode, which expressed itself as a peak in the 


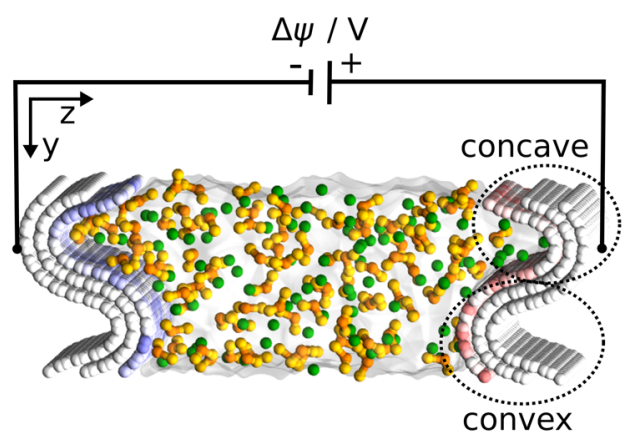

FIG. 1. Example of a simulated model supercapacitor in which concave areas form nanoscopic slitlike pores. Cations are yellow and orange, anions green, and the solvent is transparent. Electrode atoms are colored according to their charge (positive red, negative blue, and white close to zero). Table I lists concave and convex radii of the systems investigated.

differential capacitance. Such a potential-induced phase transition of the double layer at flat electrodes has also been observed experimentally [28,41,42]. Using CONP simulations, we have previously demonstrated the influence of curved electrodes on the differential capacitance, and found correlations between the electrode curvature and the number densities of the electrolyte components as well as the orientation of the ions [9]. In this work, we employ the histogram reweighting approach to estimate free energies of the electric double layer formation which ultimately allows us to correlate the charge fluctuations with structural changes at the electrode-electrolyte interface. We show that convexly or concavely curved surfaces contribute differently to the capacitance depending on the voltage, and somewhat counterintuitively, that convexly shaped surfaces are dominant at higher voltages. To assess the relative contributions of convex and concave surfaces to charge storage, we perform nanoscopic simulations at different applied potentials utilizing a coarse-grained model of a $1.5 M$ electrolyte solution, containing 1-butyl-3methylimidazolium hexafluorophosphate $[\mathrm{BMI}]\left[\mathrm{PF}_{6}\right][43]$ in acetonitrile (ACN) [44], between two rippled electrodes. It is worth noting that the chosen force fields have already been applied to carbon electrolyte interfaces, including porous carbons, and showed good agreement with all-atom models and experiments for a range of structural and dynamic properties $[45,46]$. Three systems with different curvature radii of the convex and concave electrode parts are simulated. The system shown in Fig. 1 exemplifies the structural features of the electrodes, which are summarized in Table I (radii are related to the layer in direct contact with the electrolyte). For simplicity, we introduce the notation $\chi_{5}, \chi_{7}$, and $\chi_{9}$ to distinguish the systems (see Supplemental Material [47] for a discussion on the notation).

While a linear increase in surface charge at (small) voltages can be expected at the dilute limit [54,55], which is roughly followed by the flat electrodes, rippled electrodes
TABLE I. Radii of the concave and convex parts of the carbon electrode in reference to the chirality of a corresponding nanotube (see Supplemental Material [47], Fig. S1). Radii are related to the layer in direct contact with the electrolyte, $d_{\text {sep }}$ is the distance between concave slitlike pores and $h$ their approximate depth. All values are given in $\AA$.

\begin{tabular}{lrccc}
\hline \hline System & $R_{\text {conv }}$ & $R_{\text {conc }}$ & $d_{\text {sep }}$ & $h$ \\
\hline$\chi_{5}$ & 9.8 & 3.6 & 29.7 & 15.6 \\
$\chi_{7}$ & 10.9 & 4.7 & 32.6 & 18.5 \\
$\chi_{9}$ & 12.0 & 5.8 & 37.1 & 21.3 \\
\hline \hline
\end{tabular}

show some waviness in the relationship between surface charge density and applied potential, as shown by the corresponding free energy surfaces in Fig. 2. In general, the free energy landscape of rippled electrodes is more nonlinear and steeper in comparison to that of flat electrodes [cf. Fig. 2(d)]. The (local) confinement of the undulated electrodes, especially in the concave slitlike parts, most probably restricts in part the ion diffusion, resulting in a rougher free energy landscape. This hypothesis is supported by more nonlinear free energies of the ion number densities (cf. Fig. S3) and remarkably different favorable $\mathrm{BMI}^{+}$orientation angles (cf. Fig. S5).

Utilizing the charge-fluctuation-relationship of Limmer et al. [8], connecting the differential capacitance to the total electrode charge variance $\left\langle\left(\delta Q^{ \pm}\right)^{2}\right\rangle$ in Eq. (1), differential capacitances for different curvatures are summarized in the Supplemental Material [47], Fig. S2. The capacitance for the flat electrodes is $2.46 \mathrm{~F} \mathrm{~cm}^{-2}$ on average which is similar to the value of $2.3 \mathrm{~F} \mathrm{~cm}^{-2}$ obtained using a linear approximation in a previous work by Merlet et al. [56]and is in fact in the error range specified there. We note that charge fluctuations obtained from a reweighting scheme give a more reliable and robust estimate due to an increased sampling. Larger variations of the differential capacitance with the applied potential are observed for rippled electrodes compared to flat ones, as expected from the free energies shown in Figs. 2, S3, and S5.

One might think that high capacitance peaks are associated with a phase transition in the EDL (i.e., unstable intermediate states) as have been observed previously at flat electrodes [57]. On the other hand, following the argumentation of Li et al. [33], more pronounced variations could also be related to changes within the double layer composition. In the following, an attempt is made to rationalize the observed high capacitance peaks on the basis of free energy differences. Fluctuations in the double layer composition will result in charge fluctuations in the electrode surface. Both ions are typically found in the EDL at both electrodes, but the more complex structure of $\mathrm{BMI}^{+}$ compared to the virtually spherical shape of $\mathrm{PF}_{6}^{-}$makes the electrode where more $\mathrm{BMI}^{+}$adsorbs more interesting. Moreover, previous results for flat electrodes have shown that the capacitances of the positive and negative electrodes 


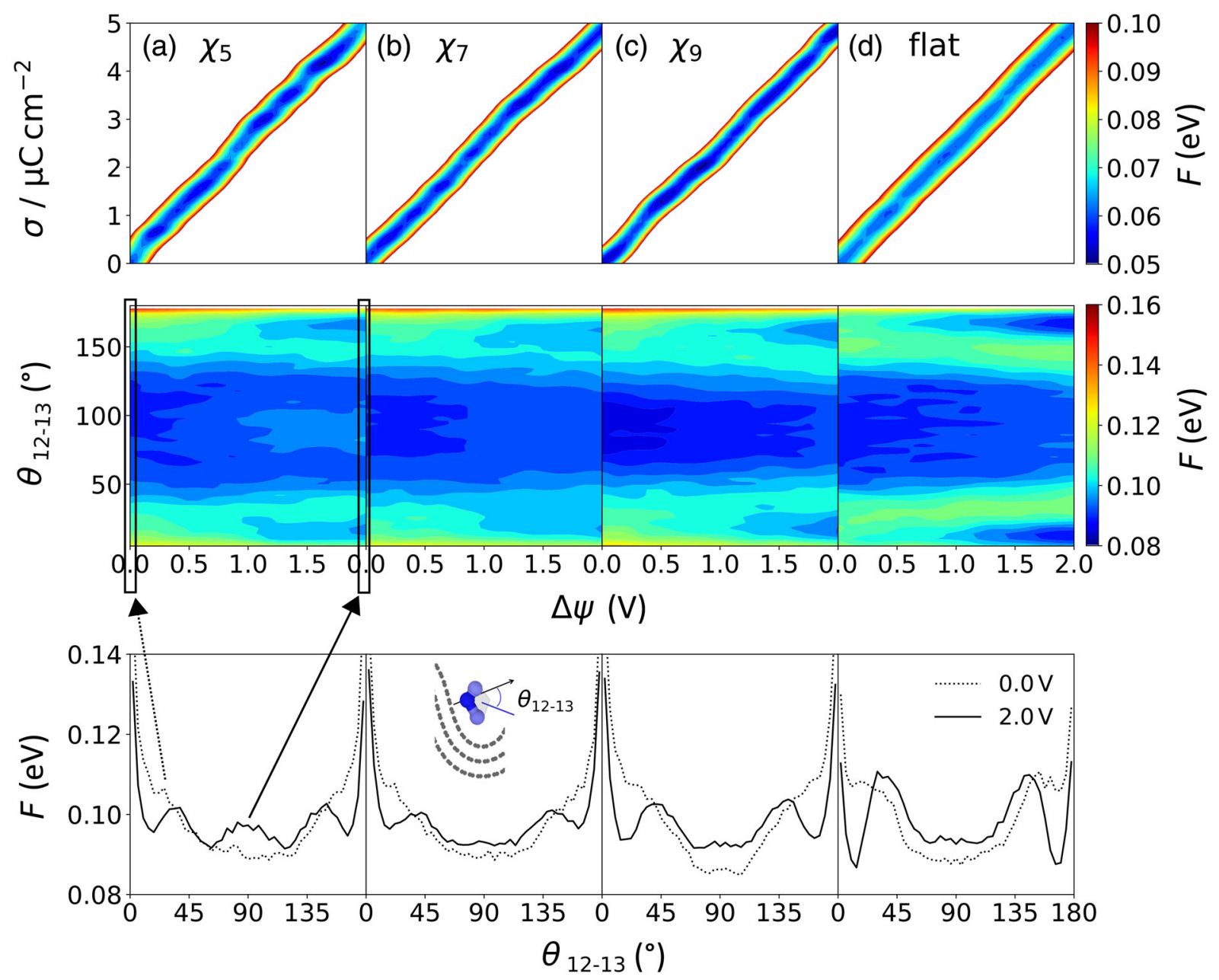

FIG. 2. (Top) free energies of the surface charge for rippled (negative) electrodes $\chi_{5}$ (a), $\chi_{7}$ (b), $\chi_{9}$ (c), and a flat electrode (d) with respect to the applied potential. Energies greater than $0.1 \mathrm{eV}$ are white. (Middle) free energies of the $\mathrm{BMI}^{+}$orientation angle $\theta_{12-13}$ (see inset in bottom row) with respect to the applied potential $\Delta \psi$. (Bottom) slices from the $\mathrm{BMI}^{+}$orientation angle free energy surfaces at 0 (dashed line) and $2 \mathrm{~V}$ (solid line).

are equal for this electrolyte, i.e., no electrode is limiting the total capacitance [56]. For the simulations done here, the relative difference between the differential capacitances of the two electrodes is typically less than $1 \%$ for the applied potentials. Consequently, the discussion concentrates in the following only on the negative electrode. As previously observed in Seebeck et al. [9], number density fluctuations of the imidazole group in the EDL with respect to different applied electric potentials, shown in Fig. S3, correlate to features in the differential capacitance of Fig. S2 [47]. We note that following the same published work [9], the EDL thickness was found to be mostly independent of the voltage and as such is not expected to play a role here. Interestingly, peaks in the differential capacitance coincide with barriers between local minima in the free energy landscape of the imidazole number density (given in Fig. S3) - where corresponding charge carrier fluctuations in the EDL are highest. As anticipated, the anion density decreases quite abruptly already at low voltages, probably because of strong electrostatic repulsions, and decreases much less afterwards.

Merlet et al. [57] correlated a capacitance peak at $0.9 \mathrm{~V}$ for a pure ionic liquid system with a phase transition in the ionic layer associated with a reorientation of $\mathrm{BMI}^{+}$at the electrode-electrolyte interface. Analyzing the free energy differences associated with such rearrangement at flat electrodes, as given in the bottom right panel of Fig. 2, a similar but less pronounced reorientation of $\mathrm{BMI}^{+}$ starting around $1.0 \mathrm{~V}$ from a minima at $90^{\circ}$ to $10^{\circ}$ (or structurally equivalent at $170^{\circ}$ ) is observed for an organic electrolyte. We suspect the solvent to be responsible for the less pronounced free energy changes associated with the reorientation compared to the results of Merlet et al. [57]. Indeed, ion-ion correlations are stronger in pure ionic liquids than in solutions with intermediate ionic concentrations [58-60]. Rippled electrodes show a similar but even less pronounced signal and a delayed development of a second free energy basin around $10^{\circ}$ (or $170^{\circ}$ ). 
The electrode undulation appears to inhibit some of the more favorable ion orientations observed for flat graphite at potentials below $1.5 \mathrm{~V}$. Interestingly, for the electrode with the smallest concave radius, a splitting of the free energy basin at $90^{\circ}$ into two, with now a small barrier at $90^{\circ}$, is observed for potentials above $1.5 \mathrm{~V}$. In summary, number density fluctuations are more likely to cause the capacitance peaks at lower voltages than a phase transition of the ions, and vice versa at higher electric potentials.

To obtain more information about the influence of individual electrode atoms and ultimately disentangle the convex and concave contributions to the differential capacitance, the charge fluctuations of each carbon atom in the negative electrode are used to calculate a charge-charge covariance matrix $\mathbf{K}_{q q}^{-}$. The differential capacitance of the electrode can be decomposed into a sum of individual atomic capacitances, $C_{q}^{ \pm}$, in reference to a parallel circuit of capacitors, plus a contribution from covariances of electrode charges, $C_{q q}^{ \pm}$:

$$
C_{\mathrm{diff}}^{ \pm}=\beta\left\langle\left(\delta Q^{ \pm}\right)^{2}\right\rangle=C_{q}^{ \pm}+C_{q q}^{ \pm},
$$

where $\beta=1 /\left(k_{B} T\right)$ with $k_{B}$ the Boltzmann constant and $T$ the temperature. $\left\langle\left(\delta Q^{ \pm}\right)^{2}\right\rangle$ is the variance of the total charge $Q^{ \pm}$on the positively or negatively charged electrode. See Supplemental Material [47], Eq. (S7) and the related explanation for a more detailed derivation. $C_{q}^{ \pm}$is given by

$$
C_{q}^{ \pm}=\sum_{i=1}^{M^{ \pm}} C_{\mathrm{diff}, i}^{ \pm}=\beta \sum_{i=1}^{M^{ \pm}}\left\langle\left(\delta q_{i}^{ \pm}\right)^{2}\right\rangle
$$

where $q_{i}^{ \pm}$represents the charge of the $i$ th atom in the positive or negative electrode (indicated by the coloring of the electrode atoms in Fig. 1) and $\left\langle\left(\delta q_{i}^{ \pm}\right)^{2}\right\rangle$ its variance $\operatorname{var}\left(q_{i}^{ \pm}\right)$. The contributions from electrode atom charge covariances are given by

$$
C_{q q}^{ \pm}=2 \beta \sum_{i=1}^{M^{ \pm}-1} \sum_{j=i+1}^{M^{ \pm}}\left\langle\left(q_{i}^{ \pm}-\left\langle q_{i}^{ \pm}\right\rangle\right)\left(q_{j}^{ \pm}-\left\langle q_{j}^{ \pm}\right\rangle\right)\right\rangle ;
$$

$M^{ \pm}$runs in both cases over the electrode atoms in either the negative or positive electrode. Free energy estimates for each individual electrode atom charge at a given electric potential are obtained by reweighting the trajectory using the individual electrode atom charge as the collective variable. Expectation values of $\left\langle q_{i}^{ \pm}\right\rangle$are calculated from the free energies and inserted into Eqs. (2) and (3) which yield a continuous function of $C_{q}^{ \pm}$and $C_{q q}^{ \pm}$with respect to the potential, as shown in Fig. 3. In general, $C_{q}^{ \pm}$has an order of magnitude higher impact on the capacitance than the empty capacitance $C_{\text {diff }}^{\text {empty }}$, given in Fig. S6, but is in comparison to the correlation capacitance $C_{q q}^{ \pm}$still small. Quite remarkably, however, a significantly higher $\operatorname{var}\left(q_{i}^{-}\right)$is observed in

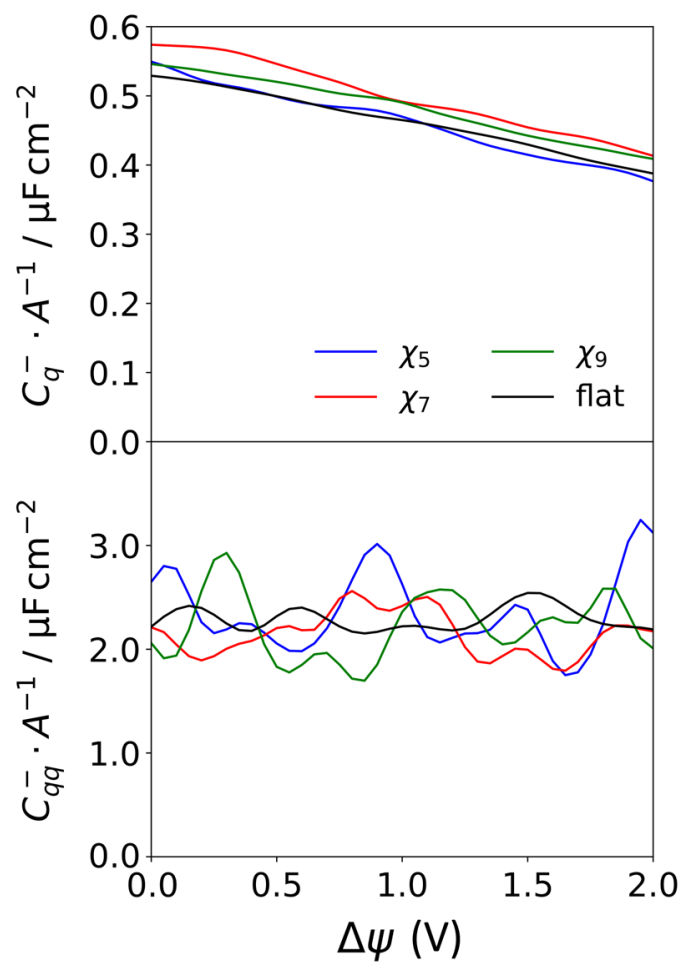

FIG. 3. Trace and sum of off-diagonal terms of the negative electrode covariance matrix $\mathbf{K}_{q q}^{-}$, i.e., $C_{q}^{-}$and $C_{q q}^{-}$, respectively, with respect to the applied potentials.

Fig. 4(b) for electrode atoms in the convex part of the $\chi_{5}$ system indicating thus a higher contribution of convex parts to the differential capacitance.

A measure of the relative contribution of each electrode atom to the total differential capacitance, as given in Fig. 4(c) for the $\chi_{5}$ electrode, is obtained by a summation of all values in each row (or column) of the charge-charge covariance matrix. At low potentials a high contribution of $C_{q q}$ is observed at turning points between convex and concave areas. In contrast, at potentials over $1.75 \mathrm{~V}$ a strong contribution of convex parts to $C_{q q}$ [indicated by high values of $\left.\sum_{j} \operatorname{cov}\left(q_{i}, q_{j}\right)\right]$ is observed-in agreement with the previous assumption of an unstable $\mathrm{BMI}^{+}$orientation associated with high charge variations at convex electrode parts. By confining them in slitlike parts, concave electrode surfaces partly inhibit the motion of ions, while they are more mobile on convex parts.

To summarize, interestingly, the charge-charge covariance matrix contains information about the individual contributions to the differential capacitance, which are represented by individual atomic capacitances in a parallel equivalent circuit, as well as contributions from correlations between atomic electrode charges. Rippled electrodes, even with rather large convex curvatures, showed significantly higher capacitance peaks compared to flat electrodes - with huge implications as ideally flat graphite electrodes, often used as a model system, are rarely found in relevant 


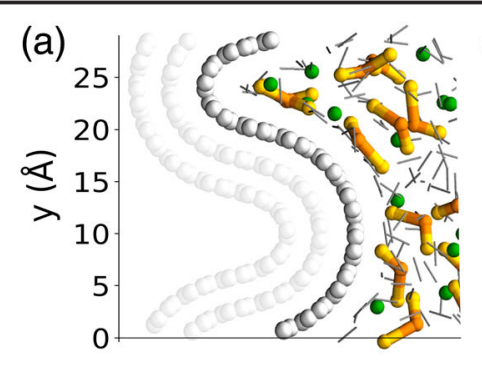

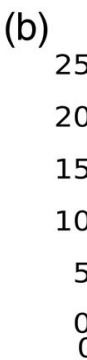

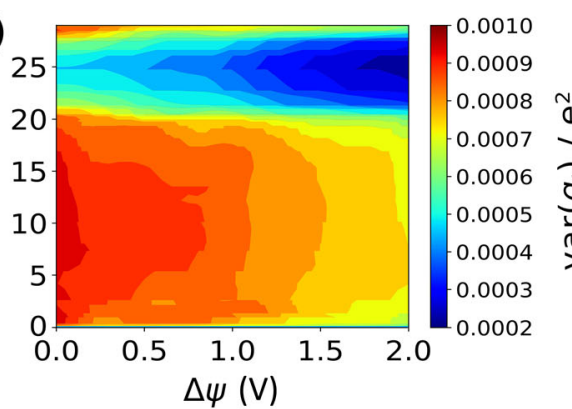

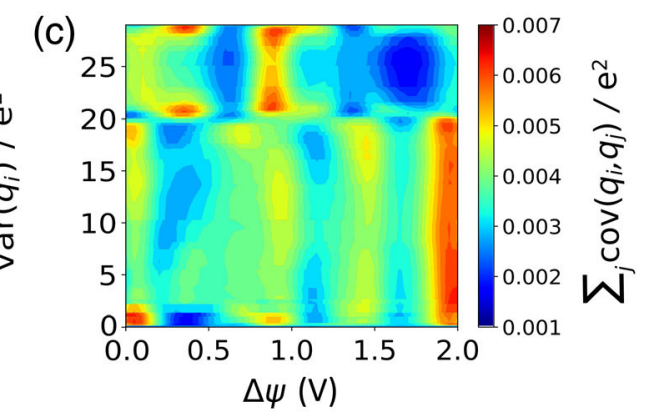

FIG. 4. Graphical illustration of the negative $\chi_{5}$ electrode atoms (white; layers which do not contribute to results are transparent), $\mathrm{BMI}^{+}$(yellow and orange), $\mathrm{PF}_{6}^{-}$(green), and $\mathrm{ACN}$ (denoted by gray lines) (a) to allow a direct identification of convex and concave areas in the variance and covariance contour plots shown in (b) and (c). Charge variances (b) and covariances (c) are averaged over all atoms with the same $x$ coordinate omitting the identifiers for the negative electrode charges.

experimental materials such as carbide-derived carbons [61] with mean curvatures similar to the ones found in our systems [62]. However, the applied voltage is decisive for whether a high curvature leads to a high capacitance. This new approach for analyzing charge fluctuations, contradicts some of our earlier findings from Seebeck et al. [9], where concave parts were generally found to be more important. Although a higher contribution of individual atomic capacitances is generally observed for the convex part of the electrode, the differential capacitance at low voltages is mainly dominated by changes in the electric double layer composition in the concave part of the electrode. At higher voltages, the convex part contributes increasingly more to the differential capacitance, manifested through more unstable $\mathrm{BMI}^{+}$orientations. On concave parts, on the other hand, these dynamical reorientations are inhibited-most evident at $1.9 \mathrm{~V}$ in Fig. 4(c).

The insights from this work point the way to new design principles of supercapacitors, for example, by explicitly considering the convex surfaces in amorphous carbons, CNT forests, or schwarzite [63] structures, and shed light on the unexpected charge storage mechanisms on convex electrodes that point to a promising capacitance enhancement. The approach presented in this work paves the way for future studies to disentangle the contributions from specific areas of supercapacitors to the capacitance.

We are very grateful to Benjamin Rotenberg for providing us with the original linearized WHAM method they used which enabled a comparison here to results from UWHAM. Funded by the Deutsche Forschungsgemeinschaft (DFG, German Research Foundation) - 192346071; 390794421 - SFB 986 and GRK 2462. Furthermore, the authors gratefully acknowledge financial support by the German Ministry of Education and Research in the AktivCAPs project (Grant No. 03SF0430B). This project has received funding from the European Research Council (ERC) under the European Union's Horizon 2020 research and innovation program (Grant Agreement No. 714581). *robert.meissner@tuhh.de

[1] G. Feng, R. Qiao, J. Huang, S. Dai, B. G. Sumpter, and V. Meunier, Phys. Chem. Chem. Phys. 13, 1152 (2011).

[2] L. Yang, B. H. Fishbine, A. Migliori, and L. R. Pratt, J. Am. Chem. Soc. 131, 12373 (2009).

[3] J. Huang, B. G. Sumpter, and V. Meunier, Angew. Chem. Int. Ed. 47, 520 (2008).

[4] M. Salanne, B. Rotenberg, K. Naoi, K. Kaneko, P. L. Taberna, C. P. Grey, B. Dunn, and P. Simon, Nat. Energy 1, 16070 (2016).

[5] C. Choi, D. S. Ashby, D. M. Butts, R. H. DeBlock, Q. Wei, J. Lau, and B. Dunn, Nat. Rev. Mater. 5, 5 (2020).

[6] S. Schweizer, J. Landwehr, B. J. Etzold, R. H. Meißner, M. Amkreutz, P. Schiffels, and J. R. Hill, J. Phys. Chem. C 123, 2716 (2019).

[7] S. Schweizer, R. Meißner, M. Amkreutz, K. Thiel, P. Schiffels, J. Landwehr, B. J. M. Etzold, J.-R. R. Hill, R. Meissner, M. Amkreutz, K. Thiel, P. Schiffels, J. Landwehr, B. J. M. Etzold, and J.-R. R. Hill, J. Phys. Chem. C 121, 7221 (2017).

[8] D. T. Limmer, C. Merlet, M. Salanne, D. Chandler, P. A. Madden, R. Van Roij, and B. Rotenberg, Phys. Rev. Lett. 111, 106102 (2013).

[9] J. Seebeck, P. Schiffels, S. Schweizer, J.-R. Hill, and R. H. Meißner, J. Phys. Chem. C 124, 5515 (2020).

[10] D. L. Z. Caetano, S. J. de Carvalho, G. V. Bossa, and S. May, Phys. Rev. E 104, 034609 (2021).

[11] M. Janssen, Phys. Rev. E 100, 042602 (2019).

[12] P. Malgaretti, M. Janssen, I. Pagonabarraga, and J. M. Rubi, J. Chem. Phys. 151, 084902 (2019).

[13] J. Vatamanu, M. Vatamanu, O. Borodin, and D. A. Bedrov, J. Phys.: Condens. Matter 28, 464002 (2016).

[14] S. Bi, H. Banda, M. Chen, L. N. Niu, M. Chen, T. Wu, J. Wang, R. Wang, J. Feng, T. Chen, M. Dinca, A. Kornyshev, and G. Feng, Nat. Mater. 19, 552 (2020).

[15] S. Kondrat, C. R. Pérez, V. Presser, Y. Gogotsi, and A. A. Kornyshev, Energy Environ. Sci. 5, 6474 (2012).

[16] L. Scalfi, D. T. Limmer, A. Coretti, S. Bonella, P. A. Madden, M. Salanne, and B. Rotenberg, Phys. Chem. Chem. Phys. 22, 10480 (2020).

[17] S. Kondrat, P. Wu, R. Qiao, and A. A. Kornyshev, Nat. Mater. 13, 387 (2014). 
[18] Y. He, R. Qiao, J. Vatamanu, O. Borodin, D. Bedrov, J. Huang, and B. G. Sumpter, J. Phys. Chem. Lett. 7, 36 (2016).

[19] L. Scalfi, M. Salanne, and B. Rotenberg, Annu. Rev. Phys. Chem. 72, 189 (2021).

[20] J. I. Siepmann and M. Sprik, J. Chem. Phys. 102, 511 (1995).

[21] S. K. Reed, O. J. Lanning, and P. A. Madden, J. Chem. Phys. 126, 084704 (2007).

[22] K. Breitsprecher, K. Szuttor, and C. Holm, J. Phys. Chem. C 119, 22445 (2015).

[23] P. Wang, Y. Li, L. Wang, J. Kłos, Z. Peng, N. Kim, H. Bluhm, K. Gaskell, P. Liu, S. B. Lee, B. W. Eichhorn, and Y. Wang, EcoMat 2, e12023 (2020).

[24] M. Busch, T. Hofmann, B. Frick, J. P. Embs, B. Dyatkin, and P. Huber, Phys. Rev. Mater. 4, 055401 (2020).

[25] A. C. Forse, J. M. Griffin, C. Merlet, P. M. Bayley, H. Wang, P. Simon, and C. P. Grey, J. Am. Chem. Soc. 137, 7231 (2015).

[26] F. Endres, N. Borisenko, S. Z. E. Abedin, R. Hayes, and R. Atkin, Faraday Discuss. 154, 221 (2012).

[27] C. Müller, K. Németh, S. Vesztergom, T. Pajkossy, and T. Jacob, Phys. Chem. Chem. Phys. 18, 916 (2016).

[28] G.-B. Pan and W. Freyland, Chem. Phys. Lett. 427, 96 (2006).

[29] I. Siretanu, D. Ebeling, M. P. Andersson, S. L. S. Stipp, A. Philipse, M. C. Stuart, D. van den Ende, and F. Mugele, Sci. Rep. 4, 4956 (2014).

[30] A. Elbourne, S. McDonald, K. Voïchovsky, F. Endres, G. G. Warr, and R. Atkin, ACS Nano 9, 7608 (2015).

[31] J. M. Black, M. B. Okatan, G. Feng, P. T. Cummings, S. V. Kalinin, and N. Balke, Nano Energy 15, 737 (2015).

[32] X. Zhang, Y.-X. Zhong, J.-W. Yan, Y.-Z. Su, M. Zhang, and B.-W. Mao, Chem. Commun. 48, 582 (2012).

[33] Z. Li, G. Jeanmairet, T. Méndez-Morales, B. Rotenberg, and M. Salanne, J. Phys. Chem. C 122, 23917 (2018).

[34] R. Burt, K. Breitsprecher, B. Daffos, P.-L. Taberna, P. Simon, G. Birkett, X. S. Zhao, C. Holm, and M. Salanne, J. Phys. Chem. Lett. 7, 4015 (2016).

[35] A. P. Willard, D. T. Limmer, P. A. Madden, and D. Chandler, J. Chem. Phys. 138, 184702 (2013).

[36] D. T. Limmer, A. P. Willard, P. Madden, and D. Chandler, Proc. Natl. Acad. Sci. U.S.A. 110, 4200 (2013).

[37] A. P. Willard, S. K. Reed, P. A. Madden, and D. Chandler, Faraday Discuss. 141, 423 (2009).

[38] M. Chen, J. Wu, T. Ye, J. Ye, C. Zhao, S. Bi, J. Yan, B. Mao, and G. Feng, Nat. Commun. 11, 5809 (2020).

[39] Z. Li, G. Jeanmairet, T. Méndez-Morales, B. Rotenberg, and M. Salanne, J. Phys. Chem. C 122, 23917 (2018).

[40] Y. Matsumi, H. Nakano, and H. Sato, Chem. Phys. Lett. 681, 80 (2017).

[41] R. Wen, B. Rahn, and O. M. Magnussen, Angew. Chem. Int. Ed. 54, 6062 (2015).
[42] W.-Y. Tsai, J. Come, W. Zhao, R. Wang, G. Feng, B. P. T., S. Dai, L. Collins, and N. Balke, Nano Energy 60, 886 (2019).

[43] D. Roy and M. Maroncelli, J. Phys. Chem. B 114, 12629 (2010).

[44] D. M. Edwards, P. A. Madden, and I. R. McDonald, Mol. Phys. 51, 1141 (1984).

[45] C. Merlet, M. Salanne, B. Rotenberg, and P. A. Madden, J. Phys. Chem. C 115, 16613 (2011).

[46] E. H. Lahrar, A. Belhboub, P. Simon, and C. Merlet, ACS Appl. Mater. Interfaces 12, 1789 (2020).

[47] See Supplemental Material at http://link.aps.org/ supplemental/10.1103/PhysRevLett.128.086001 for further details on the methods used, which includes Refs. [48-50], the model generation, which includes Refs. [51-53], a more detailed analysis of the number densities and orientation of the ionic liquids in the EDL, and the calculation of the vacuum capacitances.

[48] A. P. Thompson, H. M. Aktulga, R. Berger, D. S. Bolintineanu, W. M. Brown, P. S. Crozier, P. J. in't Veld, A. Kohlmeyer, S. G. Moore, T. D. Nguyen, R. Shan, M. J. Stevens, J. Tranchida, C. Trott, and S. J. Plimpton, Comput. Phys. Commun. 271, 108171 (2022).

[49] G. Ciccotti and J. Ryckaert, Comput. Phys. Rep. 4, 346 (1986).

[50] B. W. Zhang, S. Arasteh, and R. M. Levy, Sci. Rep. 9, 2803 (2019).

[51] L.-C. Qin, Phys. Chem. Chem. Phys. 9, 31 (2007).

[52] C. de Tomas, I. Suarez-Martinez, and N. A. Marks, Carbon 109, 681 (2016).

[53] H. Los and A. Fasolino, Phys. Rev. B 68, 024107 (2003).

[54] M. Gouy, J. Phys. Theor. Appl. 9, 457 (1910).

[55] D. L. Chapman, Philos. Mag. 25, 475 (1913).

[56] C. Merlet, M. Salanne, B. Rotenberg, and P. A. Madden, Electrochim. Acta 101, 262 (2013).

[57] C. Merlet, D. T. Limmer, M. Salanne, R. van Roij, P. A. Madden, D. Chandler, and B. Rotenberg, J. Phys. Chem. C 118, 18291 (2014).

[58] B. Uralcan, I. A. Aksay, P. G. Debenedetti, and D. T. Limmer, J. Phys. Chem. Lett. 7, 2333 (2016).

[59] M. V. Fedorov and A. A. Kornyshev, Chem. Rev. 114, 2978 (2014).

[60] M. W. Thompson, R. Matsumoto, R. L. Sacci, N. C. Sanders, and P. T. Cummings, J. Phys. Chem. B 123, 1340 (2019).

[61] V. Presser, M. Heon, and Y. Gogotsi, Adv. Funct. Mater. 21, 810 (2011).

[62] M. Oschatz, P. Pré, S. Dörfler, W. Nickel, P. Beaunier, J.-N. Rouzaud, C. Fischer, E. Brunner, and S. Kaskel, Carbon 105, 314 (2016).

[63] E. Braun, Y. Lee, S. M. Moosavi, S. Barthel, R. Mercado, I. A. Baburin, D. M. Proserpio, and B. Smit, Proc. Natl. Acad. Sci. U.S.A. 115, E8116 (2018). 